\title{
INTRAHEPATIC HEMATOMA AND HAEMOBILIA AFTER PERCUTANEOUS LIVER BIOPSY: A CLINICAL CASE REPORT
}

\author{
Goran Sarafiloski, \\ Mimi R. Marinova, \\ Pencho T. Tonchev ${ }^{1}$ \\ Department of Gastroenterology, \\ Medical University - Pleven \\ ${ }^{1}$ Department of Surgery, \\ Medical University - Pleven
}

\author{
Corresponding Author: \\ Goran Sarafiloski \\ Department of Gastroenterology, \\ Medical University - Pleven \\ 1, St. Kl. Ohridski Str. \\ Pleven, 5800 \\ Bulgaria \\ e-mail:dr_gorann@yahoo.com
}

Received: March 31, 2021

Revision received: May 10, 2021

Accepted: July 08, 2021

\section{Summary}

Assessing the severity of liver disease and predict the response to treatment in clinical practice requires the determination of the degree of inflammation progression and liver fibrosis. Percutaneous liver biopsy is the gold standard for grading and staging liver diseases. Complications are more common in the presence of vascular liver lesions, dilation of the bile ducts, ascites, or whether examination has been performed by less experienced physicians. Bleeding after liver biopsy is considered the most common cause of severe complications. Bleeding usually presents as a subcapsular or parenchymal hematoma, free intraperitoneal hemorrhage, hemobilia, or, rarely, hemothorax. The rarest of hemorrhagic complications is hemobilia, a term used to describe bleeding in the bile ducts. Hemobilia is usually suspected when there is a drop in hemoglobin after the procedure, pain in the upper right quadrant of the abdomen, hyperbilirubinemia, and unexplained gastrointestinal bleeding. The clinical manifestations range from chronic anemia to rapid, massive bleeding with hematemesis and/or melena.

Keywords: liver biopsy, complication, intrahepatic hematoma, hemobilia, melena

\section{Introduction}

We present a patient with intrahepatic hematoma and hemobilia after liver biopsy performed on a chronic viral hepatitis $\mathrm{C}$ for specific antiviral treatment.

Liver biopsy (LB) plays a crucial role in the algorithms for diagnosis and treatment in patients with chronic liver disease and remains the gold standard for assessing liver pathology. To determine the severity of liver damage and predict treatment response in clinical practice, the degree of inflammation and liver fibrosis have to be evaluated. Liver biopsy is performed by various techniques - percutaneous, transjugular, and laparoscopic. The latter two manipulations are performed in the presence of relative contraindications for percutaneous liver biopsy $[1,2]$.

Absolute contraindications for liver biopsy are:

- severe hemostasis disorders, hemorrhagic diathesis;

- purulent-inflammatory changes in the abdomen, pleura, the liver itself because of the risk of spreading the infection (pleurisy, empyema, cholangitis, acute cholecystitis, subphrenic or other abscesses); 
- pustular or eczematous processes, dermatitis at the points of the planned puncture or incision;

- severe portal hypertension;

- massive ascites;

- focal processes in the liver (vascular lesions, cystic formations - echinococcal cysts, tumors);

- extrahepatic cholestasis;

- changes in the anatomy of the bile ducts;

- lack of liver dullness (small liver, severe emphysema, Chilaiditi's syndrome);

- disorders of consciousness, coma;

- mental illness in which contact with the patient is difficult.

Relative contraindications for liver biopsy include:

- heart or respiratory failure;

- hypertension;

- severe anemia;

- severe cachexia;

- obesity;

- allergy to local anesthetics;

- the disagreement of the patient.

Focal processes in the liver are diagnosed by abdominal ultrasound. [3,4]. Preliminary laboratory tests are performed - complete blood count, coagulation status, biochemistry. The patient should be informed about the whole procedure and possible complications and sign an informed consent.

According to literature data, complications after liver biopsy occur in $1-5 \%$ of patients. About $60 \%$ of complications appear within two hours after the procedure, and $96 \%$ - by the twenty-fourth hour $[4,5,6]$. The frequency of complications correlates with the diameter of the needle, the time it is held in the liver, and the depth to which the needle inserts into the liver tissue. Possible complications are pain, bleeding, damage to a neighboring organ (lung, gallbladder), or infection. Hemorrhage, biliary peritonitis, and pneumothorax are potentially fatal. Bleeding is observed at $0.32-0.35 \%$. Mortality from severe bleeding is $0.11 \%[6,7]$. It presents as a subcapsular or parenchymal hematoma, intraperitoneal hemorrhage, hemobilia, or, rarely, hemothorax.

The rarest of hemorrhagic complications is hemobilia. This term was first used in 1946 by Sandlom to describe bleeding from the bile ducts. A review of Sandblom's hemobilia cases showed that in about 50 percent of the cases, it was caused by trauma, followed by iatrogenic causes in one-third of the cases. The most common cause reported is needle biopsy of the liver $[8,9]$.

\section{Case Report}

zA 56-year-old patient with proven liver cirrhosis since 2003 based on chronic HCV infection since 1996 was diagnosed by blood donation. He had suffered several hemorrhages from esophageal varices, the first of which was in 2003. In 2005 he was operated on for hemorrhage from esophageal varices. Splenectomy was performed due to severe hypersplenism syndrome. Since then, several endoscopic ligations of the varicose veins had been performed. He received constant therapy with Propranolol 20-40 mg, depending on the values of blood pressure, as well as hepatoprotectors and diuretics.

The patient was admitted for liver biopsy for staging and grading the disease, and a protocol was to be prepared for specific antiviral treatment following the requirements of the National Health Insurance Fund.

Results from laboratory tests were as follows: revealed tests: leukocytes $-3.9 \times 10^{9} / 1$, hemoglobin - $127 \mathrm{~g} / 1$, platelets - $173 \times 10^{9} / 1$, ALT - 81UI/1, AST - 114UI/1, GGTP - 98UI/1, $\mathrm{AF}-72 \mathrm{UI} / 1$, total bilirubin $-23 \mathrm{mmol} / 1$, direct bilirubin $-13 \mathrm{mmol} / 1$, prothrombin time $-71.1 \%$, INR - 1.23.

Abdominal ultrasound revealed diffuse structural disorders of the liver parenchyma. There was no evidence of free intraperitoneal fluid.

The patient underwent LB without complications. After the manipulation, CBC showed - leukocytes $-4.8 \times 10^{9} / 1$, hemoglobin $126 \mathrm{~g} / \mathrm{l}$, platelets $-173 \times 10^{9} / 1$.

Six days after the liver biopsy, the patient reported severe colic pain in the right hypochondrium, nausea, vomiting, and black stools.

The results from the control laboratory tests were as follows: leukocytes $-10.8 \times 10^{9} / 1$, hemoglobin - 103g/l, platelets - 184x10 $/ 1$, ALT - 65UI/1, AST - 78UI/1, GGTP - 226UI/1. Total bilirubin - 195mmol/, direct bilirubin $179 \mathrm{mmol} / 1$, prothrombin time -70.5 , INR - 1.2.

Abdominal ultrasound showed enlargement 
of the liver with a non-homogeneous structure of the parenchyma. The lateral part of the right lobe next to the gallbladder, an anechogenic zone with dimensions $127 / 55 \mathrm{~mm}$, with hyperechogenic areas inside it (hematoma) was visualized. There was a minimum amount of free fluid in the abdomen.

CAT confirmed the presence of a hematoma.

On gastroscopy, varicose veins of the esophagus, blood in the lumen of the stomach and duodenum were seen.

After consultation with a surgeon and resuscitator, a decision was made for surgical treatment of the patient in the Clinic of Abdominal and Visceral Surgery. Exploratory laparotomy revealed the presence of a hematoma located between the porta hepatis, the lesser curvature of the stomach, and the gallbladder. The hematoma (about $300 \mathrm{ml}$ of old, hemolyzed blood) was evacuated. The hematoma cavity was treated with HaemoCer ${ }^{\mathrm{TM}}$, and three hemostatic sponge strips were inserted. Bleeding in the liver was also found, which was sewn to achieve hemostasis, and a thorough lavage with aspiration was performed. A subhepatic drain was placed.

Postoperatively, therapy was continued in the Intensive Care Unit with saline water resuscitation, hemostasis, blood transfusion, antibiotics, and a nil per os diet. Despite the therapy, the patient's condition did not improve, the hemodynamic status aggravated, and melena persisted. On the 4th day after the operation, a gastroscopy was performed, and clear blood was found postbulbarly in the duodenum, and the papilla of Vaters was covered with fresh coagulum. It was diagnosed as hemobilia.

The patient was referred to a specialized medical institution for more precise diagnostics and highly specialized medical procedures. Selective angiography established communication between a blood vessel and a bile duct. Selective embolization of the segmental hepatic artery was performed to control the bleeding.

At follow-up examinations after the intervention, the patient felt well. The histological result of the liver biopsy verified the diagnosis of chronic hepatitis with high activity (A3F3), Metavir score was 16 points. Antiviral therapy for hepatitis $\mathrm{C}$ was administered, and the virus was cleared of his body.

\section{Discussion}

Liver biopsy is an invasive and, therefore, potentially dangerous procedure, widely used for diagnosis and evaluation of various liver diseases, including chronic HCV infection. Complications are observed in $1-5 \%$ of the cases. Therefore, it is only in a hospital setting.

Hemorrhage and biliary peritonitis are detected in the first 4 hours after the procedure. Delayed bleeding occurs within 15 days after biopsy [10], and $23 \%$ of the patients are diagnosed with subcapsular or intrahepatic hematoma in the first 24 hours after the procedure using abdominal ultrasound. These hematomas are usually small and do not lead to serious hemodynamic disorders $[6,11]$.

Most cases of fatal bleeding, especially hemoperitoneum, result from perforation of a vessel from the portal or hepatic blood circulation. It can also occur because of a rupture of the liver when the patient takes a deep breath during the biopsy [11]. Hemoperitoneum occurs in $0.32 \%$ of the cases and clinically manifests with abdominal pain, hypotension, and tachycardia. It may be diagnosed with abdominal ultrasound or computed tomography [8].

Hemobilia is suspected in patients with a decrease in hemoglobin level after the procedure and the presence of pain in the upper right quadrant of the abdomen, changes in vital signs with decreased blood pressure and increased heart rate, high bilirubin levels, and unexplained gastrointestinal bleeding, usually during the first five days after the biopsy $[4,6,14,15,16]$. More often, the manifestations are atypical, such as ascending cholangitis, cholestasis without jaundice, melena, hematemesis, pancreatitis, or even cholecystitis. Bleeding can lead to the formation of clots in various parts of the bile duct system and obstruction of specific sites, leading to the manifestations described above. Bleeding is often intermittent and difficult to visualize with standard endoscopes with frontal optics [16]. It is usually of arterial origin but may be venous in patients with portal hypertension. The biopsy can be the reason for forming a hematoma or pseudoaneurysm in the bile ducts. Dissolution of a clot may lead to delayed bleeding. Acute bleeding is caused by concomitant perforation of the intrahepatic bile ducts and blood vessels [14]. The clinical manifestations range from chronic 
anemia to acute bleeding with hematemesis, melena, and hematochezia [16].

Doppler ultrasound can identify the needle $[8,19]$, the gallbladder, hepatic hematomas, and the presence of free intraabdominal fluid. Computed tomography and magnetic resonance imaging are performed to confirm the ultrasound finding. In cases of severe bleeding, the method of choice is selective angiography. It has both diagnostic and therapeutic value, determining the source of bleeding and suggesting a therapeutic approach - embolization or balloon occlusion of the affected segmental artery. The first angiographic embolization was described in 1976. Surgical intervention is indicated if hemodynamic instability persists despite active resuscitation. The first successful hepatic resection of the liver was performed in 1956 $[16,17,18]$.

\section{Conclusions}

Percutaneous liver biopsy is a standard method for staging and grading liver disease. Bleeding after liver biopsy presents as subcapsular or parenchymal hematoma, hemoperitoneum, or hemobilia. The diagnosis of hemobilia after liver biopsy is suspected in cases with a decrease in hemoglobin, the presence of abdominal pain, hyperbilirubinemia, or unexplained gastrointestinal bleeding.

Hemobilia is a rare cause of upper gastrointestinal bleeding and manifests as haematemesis or melena. The most common cause is trauma, mostly iatrogenic. In the case of hemobilia, selective angiography and embolization of the segmental hepatic artery should be considered.

\section{References}

1. Perrault J, Mc Gill DB, Ott BJ et al. Liver biopsy: complications in 1000 inpatients and outpatients. Gastroenterology. 1978 Jan;74(1):103-6.

2. Bravo AA, Sheth SG, Chopra S. Liver biopsy. N Engl J Med. 2001;344(7):495-500.

3. Froehlich F, Lamy O, Fried M. et al. Practice and complications of liver biopsy. Results of a nationwide survey in Switzerland. Dig Dis Sci. 1993 Aug;38(8):1480-4.

4. Bravo AA, Sheth SG, Chopra S. Liver biopsy. N Engl J Med. 2001 Feb 15;344(7):495-500.
5. van Leeuwen DJ, Wilson L, Crowe DR. Liver biopsy in the mid-1990s: questions and answers. Semin Liver Dis. 1995 Nov;15(4):340-59.

6. Piccinino F, Sagnelli E, Pasquale G, Giusti G. Complications following percutaneous liver biopsy: a multicentre retrospective study on 68,276 biopsies. J Hepatol. 1986;2(2):165-73.

7. McGill DB, Rakela J, Zinsmeister AR, Ott BJ. A 21-year experience with major hemorrhage after percutaneous liver biopsy. Gastroenterology. 1990;99(5):1396-400.

8. Sandblom P. Hemorrhage into the biliary tract following trauma; traumatic hemobilia. Surgery. 1948;24(3):571-86.

9. Vlahova M, Lalev I, Sarafiloski G. Acute upper gastrointestinal tract bleeding as seen by a gastroenterologist-endoscopist. Health.bg journal, May 2016;1:20-3.

10. Jacobs WH, Goldberg SB. Statement on outpatient percutaneous liver biopsy. Digestive diseases and sciences;34(3):322-3.

11. Minuk GY, Sutherland LR, Wiseman DA et al. Prospective study of the incidence of ultrasounddetected intrahepatic and subcapsular hematomas in patients randomized to 6 or 24 hours of bed rest after percutaneous liver biopsy. Gastroenterology. 1987 Feb;92(2):290-3.

12. Mc Gill DB, Rakela J, Zinsmeister AR, Ott BJ. A 21-year experience with major hemorrhage after percutaneous liver biopsy. Gastroenterology. 1990 Nov;99(5):1396-400.

13. Chuah SY. Liver biopsy-past, present and future. Singapore Med J. 1996;37(1):86-90.

14. Lichtenstein DR, Kim D, Chopra S. Delayed massive hemobilia following percutaneous liver biopsy: treatment by embolotherapy. Am J Gastroenterol; 1992 Dec;87(12):1833-8.

15. Mahal AS, Knauer CM, Gregory PB. Bleeding after liver biopsy. West J Med. Jan;134(1):11-4.

16. Vlahova M. Acute upper gastrointestinal tract bleeding as seen by a gastroenterologistendoscopist. Monograph, Publishing Centre of Medical University - Pleven. 2020, 34-58.

17. Sandblom P, Saegesser F, Mirkovitch V. Hepatic hemobilia: hemorrhage from the intrahepatic biliary tract, a review. World J Surg. 1984;8(1):41-50.

18. Lichtenstein DR, Kim D, Chopra S. Delayed massive hemobilia following percutaneous liver biopsy: treatment by embolotherapy. Am J Gastroenterol. 1992;87(12):1833-8.

19. Dang $\mathrm{S}$, Neelima V, Atiq $\mathrm{M}$, Olden $\mathrm{KW}$, Aduli F. Hemobilia after Percutaneous Liver Biopsy Presenting as Hematochezia: A Rare Complication of a Commonly Performed Procedure. Chir Gastroenterol. 2008;24:313-5. 